\title{
Immunization for bone marrow transplant recipients
}

\author{
B Lynn Johnston $M{ }^{1}{ }^{1}$, John M Conly MD²
}

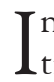
$\mathrm{n}$ a previous issue of the journal, we reviewed immunization in healthy, immunocompetent adults (1). In the present issue, we consider immunization in adults who have undergone bone marrow transplants. Bone marrow transplantation (BMT) has become a common procedure for the treatment of several types of hematological malignancies, aplastic anemia and several congenital deficiencies of the bone marrow and immune systems (2). By virtue of the pretransplant preparatory regimens, a temporary state of combined immunodeficiency occurs in all patients after transplantation, with functional recovery of humoral and cell mediated immunity potentially taking a year or longer to develop (2). Multiple components of the host protective mechanisms are impaired, including mucosal barriers, granulocytes, natural killer cells, T cells and B cells (3). Part of the immunodeficiency includes loss of the immune memory that has accumulated over many years of exposure to infectious agents and vaccines (4). While there are some data to support the transfer of memory B cells from donor to recipient, most B cells in BMT survivors have probably developed from bone marrow stem cells, which need to be stimulated to provide long term protection (3). The immunodeficient state is further modified by the use of immunosuppressive therapies, the occurrence of graft-versus-host disease (GVHD) and infection with cytomegalovirus. This immunodeficient state puts the hematopoietic stem cell recipient at increased risk for infection with a variety of pathogens, some of which are preventable by vaccine. An understanding of the level of immune responsiveness after BMT is important, not only for recognizing the risk for infection, but also for determining the optimal timing for vaccine delivery.

\section{POST-BMT IMMUNITY AGAINST VACCINE PREVENTABLE INFECTIONS}

Post-BMT antibody levels and in vitro cell mediated immune responsiveness to a variety of bacterial and viral pathogens have been studied in a number of patients, largely in the context of small, post-BMT vaccine studies. Most studies have determined that antibody levels fall after BMT and continue to fall over a number of years, despite reconstitution of the immune system. The roles of transplant source (allogeneic compared with autologous), GVHD (acute and chronic) and natural versus vaccine-induced immunity in the loss of antibody after BMT have not been completely elucidated.

In the 1980s, Ljungman et al (5) found that $77 \%$ of 48 allogeneic BMT patients and $71 \%$ of their donors were seropositive for tetanus toxoid (TT) before BMT. One year after BMT, only half of the previously immune and none of the nonimmune patients had detectable antibodies. At two years, none of the nonvaccinated patients was seropositive (5). Of note was a positive correlation between the patients' antibody levels before transplant and one year after transplant, raising the possibility of a role for immunization of patients before BMT to improve protection after BMT (5). The authors did not find that tetanus immunity was transferred to seronegative patients from their seropositive donors, but the study involved a small number of patients (5). This fall in antitetanus antibodies has been confirmed by other investigators in both allogeneic and autologous BMT patients (6-8), and one investigator also examined and found a positive correlation between pretransplant and post-transplant antibody levels (6).

${ }^{1}$ Queen Elizabeth II Health Sciences Centre and Dalhousie University, Halifax, Nova Scotia; ${ }^{2}$ Departments of Pathology and Laboratory Medicine, Medicine, and Microbiology $\mathcal{E}$ Infectious Diseases, University of Calgary, Calgary, Alberta

Correspondence and reprints: Dr Lynn Johnston, Room 5014 ACC, Queen Elizabeth II Health Sciences Centre, 1278 Tower Road, Halifax, Nova Scotia B3H 2Y9. Telephone 902-473-5553, fax 902-473-7394, e-mail ljohnsto@dal.ca and Dr John Conly, Departments of Pathology and Laboratory Medicine, Medicine, and Microbiology 8 Infectious Diseases, Department of Medicine, Room 930, 9th Floor, North Tower, 1403 29th Street Northwest, Calgary, Alberta T2N 2T9. Telephone 403-944-8222, fax 403-944-1095, e-mail jconly@ucalgary.ca 
Similar falls in protective antibody levels have been observed for poliovirus; diphtheria; measles, mumps, rubella (MMR); and Haemophilus influenzae type b (Hib). In one study, none of the seven evaluable patients had protective antibody levels against all poliovirus types one year after allogeneic BMT, although $82 \%$ had immunity before transplant (9). For diphtheria, six of 11 (55\%) had immunity at one year, compared with $64 \%$ before transplant (9). All patients with protective antibodies one year later had been immune before their BMTs and received marrow from a seropositive donor. While another study found that $67 \%$ of 55 patients had immunity to all poliovirus types one year after allogeneic BMT, approximately half of the seropositive patients had at least a four-fold decrease in antibody levels during that time (10). Two seronegative patients who had seropositive donors seroconverted in the year after BMT, but their long term protection in the absence of postBMT immunization was not evaluated (10). As noted previously, while donor B cells may provide early immune memory, it is not considered to be of long duration $(9,10)$. For autologous BMT patients, Pauksen et al (11) found that a similar percentage lost immunity to poliovirus at one year, as did allogeneic recipients. Beyond that time, however, there were fewer who lost antibodies, suggesting that patients are more likely to have residual memory cells after autologous BMT than after allogeneic BMT (11). The presence of acute GVHD was associated with a greater decrease in protective antibody against poliovirus in one study, but could not be demonstrated for chronic GVHD (12).

In two studies, only $6 \%$ to $16 \%$ of adults had antibody levels that would be considered protective against Hib six months after BMT, compared with $27 \%$ to $59 \%$ before BMT $(9,13)$. In a cohort (57 patients) of primarily paediatric, allogeneic BMT recipients, among those who were seropositive at the time of transplant, $51 \%$ had retained antibodies to measles, $42 \%$ to mumps and $76 \%$ to rubella two years later, with no differences noted according to type of pretransplant immunity (vaccine versus natural infection) or GVHD (14). On the other hand, another study found that almost $50 \%$ of children (nine of 19 ) but only $2 \%$ of adults (one of 44) became seronegative to measles after BMT (15). The study also found that a significantly higher proportion of patients who had vaccine-induced immunity became seronegative than did patients with natural immunity (15). This suggests that natural immunity is more robust than vaccine-induced immunity.

\section{POST-BMT VACCINATION}

In view of the studies demonstrating loss of protective antibodies in patients who have undergone BMT, a number of investigators have examined responses to immunization in this group of patients. Most studies were nonrandomized, included small numbers of patients and utilized different immunization schedules. It is, therefore, difficult to make comparisons between different regimens and patient groups. Furthermore, due to the rarity of most of these infections in adults, outcomes in terms of occurrence of infection have not been evaluable. However, with the exception of pneumococcal immunization, responses to vaccines in patients after BMT are generally good. Receiving more than one vaccine dose has been consistently associated with a better antibody response, in support of the theory that $\mathrm{B}$ cell immunity derives largely from donor marrow stem cells rather than memory cells $(5,10-12,16$ 19). There is little evidence to support one dosing schedule over another $(6,7,12,13,16,17)$. For tetanus and polio vaccines, there have been no differences noted between those with and without GVHD in terms of response rate $(5,6)$, although those without GVHD tend to have higher antibody levels $(6,12)$. Likewise, there is no apparent difference between autologous and allogeneic BMT recipients in response to vaccine (19) and between those who receive peripheral blood stem cells rather than bone marrow (20). On the other hand, acute and chronic GVHD did have an impact on response to pneumococcal polysaccharide vaccine, a poor immunogen (13), as did early vaccination after transplantation and corticosteroid therapy (21). Although involving small numbers of patients, none of the studies reported serious adverse events associated with immunization and most did not even comment on the frequency of minor reactions (Table 1 ).

Improving the response of patients to immunization after BMT is clearly a goal for these patients. A positive association between pretransplantation and post-transplantation antibody levels has already been noted. Although findings have not been replicated by all investigators, some have found that donor antibody status is associated with the response to vaccine, suggesting at least the short term adoption of memory cells from the donor $(6,13)$. This observation is in keeping with an early study demonstrating that B cells synthesizing antibodies against diphtheria and TT could be transferred to BMT recipients by immunizing donors before marrow harvest (22). Immunizing recipients and/or donors before BMT has been explored as a method of improving response to vaccination. In a study comparing 12 patients immunized with Hib-conjugate, TT and pneumococcal polysaccharide vaccines before bone marrow harvest to 41 nonimmunized recipients, there was a trend to higher response rate and antibody levels to Hib and TT, but not against Streptococcus pneumoniae (23). In a similar study, allogeneic BMT recipients were allocated to one of two groups where the donor was or was not immunized before bone marrow harvest. Patients whose donors were immunized with Hib-conjugate vaccine and TT had higher antibody levels after transplantation and immunization than did patients with unimmunized donors (24). The authors suggested that recipient and/or donor immunization before bone marrow harvest and transplantation may be a strategy to prevent these and other infections in BMT patients $(23,24)$.

Immunization with an investigational inactivated varicella vaccine has been examined for its ability to reduce morbidity due to varicella-zoster reactivation after BMT. In a study comparing single and three-dose regimens (begin- 
TABLE 1

Immunization trials of patients who have undergone bone marrow transplant (BMT)

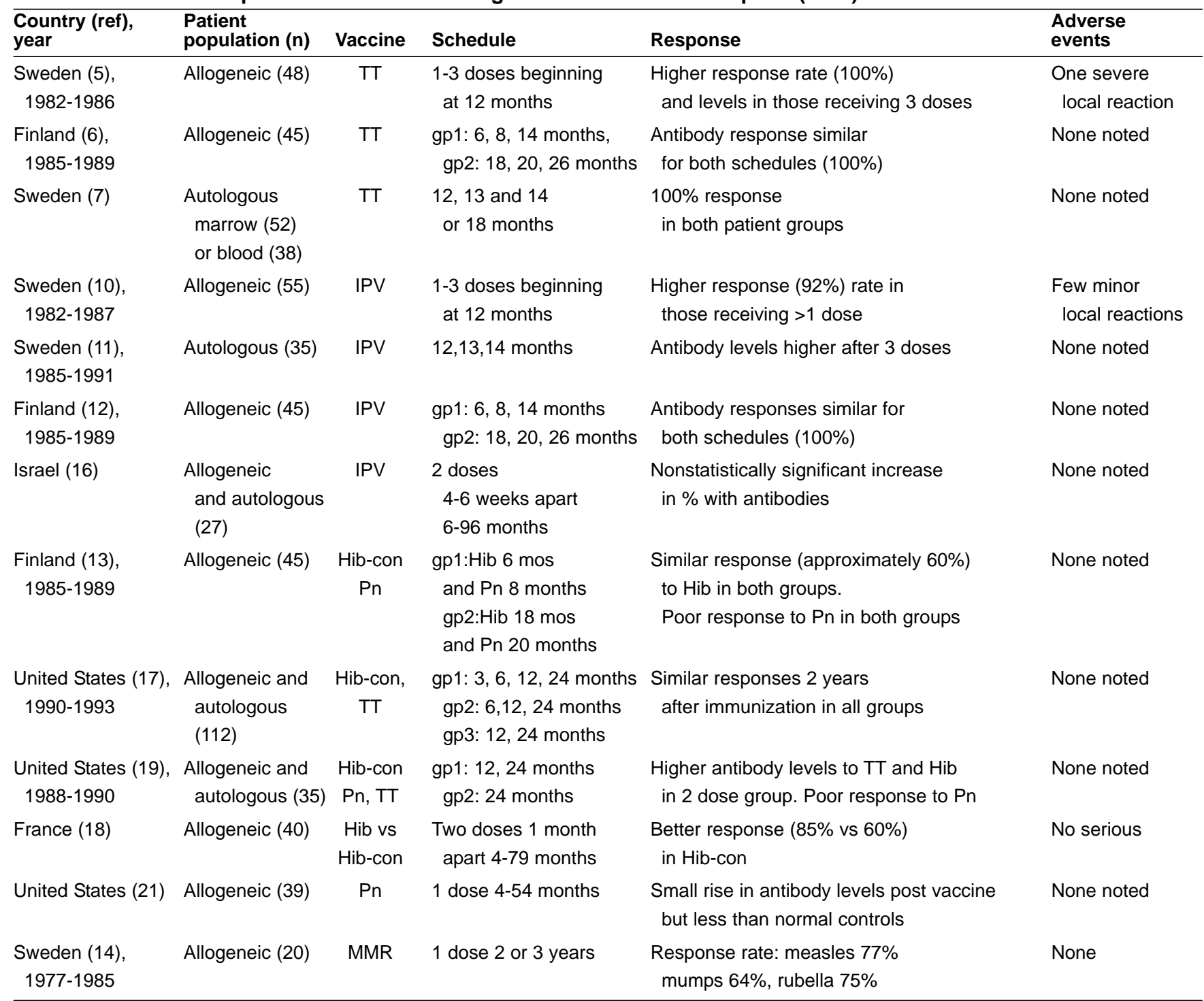

Gp Group; Hib Haemophilus influenzae type b polysaccharide vaccine; Hib-con Haemophilus influenzae type b conjugate vaccine;

IPV Inactivated poliovirus vaccine; MMR Measles, mumps, rubella vaccine; Pn Pneumococcal polysaccharide vaccine; TT Tetanus toxoid

ning one month after BMT) to unvaccinated controls, the three-dose regimen was associated with less severe zoster (25). Patients who received the three-dose regimen also had earlier recovery of $\mathrm{T}$ cell responsiveness to varicella zoster antigen than did nonimmunized controls (25). Employing the concept that pre-BMT immunization may enhance overall vaccine response, a four-dose inactivated varicella-zoster virus vaccine regimen was studied in lymphoma patients after autologous BMT. The first dose of vaccine was given two to 34 days before BMT. In contrast to the earlier study, there were no differences in severity of zoster between immunized and nonimmunized patients, but there was less zoster $(13 \%$ versus $33 \%, \mathrm{P}=0.01)$ in the vaccine group than in the control group (26). Vaccine recipients also had earlier reconstitution of $\mathrm{T}$ cell response to varicella-zoster antigen than did the controls (26). This raises promise that immunization may be effective in accelerating the recovery of cellular immunity to other herpes viruses, including cytomegalovirus (26).

\section{RECOMMENDATIONS FOR VACCINATION AFTER BMT}

The recent update of the Canadian Immunization Guidelines (27) has a section dealing with immunization in immunocompromized patients, including BMT patients. The authors acknowledge that while there is a good theoretical basis for providing immunization, there are no conclusive data to support the best schedule, the need for boosters, efficacy in disease reduction or cost benefit. Diphtheria and TT, inactivated poliovirus, Hib, influenza 


\begin{tabular}{l} 
TABLE 2 \\
Centers for Disease Control and Prevention \\
recommendations for immunization in hematopoietic \\
stem cell transplant recipients \\
\begin{tabular}{ll} 
Vaccine & Schedule \\
\hline 23-valent pneumococcal & 12 and 24 months \\
polysaccharide & \\
Haemophilus influenzae & 12,14 and 24 months \\
type b conjugate & \\
Varicella zoster virus & Contraindicated \\
Influenza & Yearly lifelong resuming six \\
& months after hematopoietic \\
Tetanus-diphtheria toxoid & stem cell transplant \\
Inactivated polio & 12,14 and 24 months \\
Hepatitis B & 12,14 and 24 months \\
Hepatitis A & 12,14 and 24 months \\
Meningococcal & Routine administration not \\
Measles, mumps, rubella & recommended \\
& Routine administration not \\
& recommended \\
& 24 months if \\
immunocompetent
\end{tabular} \\
\hline
\end{tabular}

Data from reference 4. Hematopoietic stem cell transplant (HSCT) recipients are considered immunocompetent at 24 months after HSCT if they are not on immunosuppressive therapy and do not have graft-versus-host-disease

and pneumococcal vaccines are recommended routinely after BMT (27). No specific dosing schedules are given. Meningococcal and hepatitis A vaccines are recommended if indicated, and hepatitis B vaccine as per routine use, while MMR, varicella and yellow fever vaccines may be considered at 24 months if there is no immunosuppressive therapy or disease (27). Oral polio, typhoid and cholera vaccines, as well as Bacille Calmette-Guerin are contraindicated (27). The United States Eastern Oncology Cooperative Group indicated in 1994 that it is probably reasonable to consider a number of vaccines at the end of one year without making any clear recommendations (2). In their 2000 guidelines for hematopoietic stem cell transplant recipients (Table 2), the United States Centers for Disease Control and Prevention (CDC) recommended a number of vaccines and offered immunization schedules

\section{REFERENCES:}

1. Johnston BL, Conly JM. Routine adult immunization in Canada: Recommendations and performance. Can J Infect Dis 2002;13:226-31.

2. Rowe JM, Ciobanu N, Ascensao J, et al. Recommended guidelines for the management of autologous and allogeneic bone marrow transplantation. A report from the Eastern Cooperative Oncology Group (ECOG). Ann Intern Med 1994;120:143-58.

3. Storek J, Saxon A. Reconstitution of B cell immunity following bone marrow transplantation. Bone Marrow Transplant 1992;9:395-408.

4. CDC. Guidelines for preventing opportunistic infections among hematopoietic stem cell transplant recipients. MMWR 2000;49(RR-10):1-125.

5. Ljungman P, Wiklund-Hammarsten M, Duraj V, et al. Response to
(4). The CDC and Canadian guidelines are similar except that the CDC recommends that varicella-zoster vaccine use, even in those presumed to be immunocompetent, be restricted to the research setting until further information is available (4). The European Group for Blood and Marrow Transplantation recommends in its updated guidelines that all BMT patients receive TT, diphtheria toxoid, inactivated poliovirus, pneumococcal, $H$ influenzae and influenza vaccines (28). Measles and rubella immunization is considered an individual recommendation based on risk and benefit assessment with vaccination not to be given before 24 months in allogeneic recipients (28).

As with routine immunization in adults, immunization practices following bone marrow transplant may fall short of recommendations. In a 1995 survey of BMT centres in the United States, $88 \%$ of the centres provided diphtheria and TT vaccines and $73 \%$ provided inactivated poliovirus vaccine following allogeneic BMT (29). Eighty per cent of the centres offered pneumococcal vaccine and $58 \%$ offered $\mathrm{Hib}$ conjugate vaccine (29). On the other hand, fewer than $50 \%$ of programs gave two or more doses, and there were a number of different schedules reported (29). The situation may be improving. In a 1995 survey of the European Group for Blood and Marrow Transplantation centres, 69\% and $37 \%$ of the centres reported that they routinely immunized recipients of allogeneic and autologous BMTs, respectively (30). As a result of these findings, recommendations for immunization after BMT were published (30). By 1998, $95 \%$ of centres reimmunized patients against tetanus, diphtheria and poliovirus (28). Most centres used a multidose schedule, and the majority initiated immunization at least one year from allogeneic BMT (30). Similar data are not available for Canada.

While there are exceedingly few randomized controlled trials of immunization after BMT, there is a good theoretical basis for its use. Many patients will lose immunity to vaccine-preventable infections over time following BMT. Small studies have demonstrated that for most of these infections, most BMT patients will mount an immune response with vaccination. Serious adverse events due to immunization have not been noted. Recent guidelines have been published by both the CDC and National Advisory Committee on Immunization. Physicians looking after BMT patients should review their own guidelines and practices.

tetanus toxoid immunization after allogeneic bone marrow transplantation. J Infect Dis 1990;162:496-500.

6. Parkkali T, Ölander RM, Ruutu T, et al. A randomized comparison between early and late vaccination with tetanus toxoid vaccine after allogeneic BMT. Bone Marrow Transplant 1997;19:933-8.

7. Hammarström V, Pauksen K, Björkstrand B, Simonsson B, Öberg G, Ljungman P. Tetanus immunity in autologous bone marrow and blood stem cell transplant recipients. Bone Marrow Transplant 1998;22:67-71.

8. Li Volti S, Mauro L, DiGregorio F, et al. Immune status and immune response to diphtheria-tetanus and polio vaccines in allogeneic bone marrow-transplanted thalassemic patients. Bone Marrow Transplant 1994;14:225-7. 
9. Parkkali T, Ruutu T, Stenvik M, et al. Loss of protective immunity to polio, diphtheria and Haemophilus influenzae type b after allogeneic bone marrow transplantation. APMIS 1996;104:383-8.

10. Ljungman P, Duraj V, Magnius L. Response to immunization against polio after allogeneic marrow transplantation. Bone Marrow Transplant 1991;7:89-93.

11. Pauksen K, Hammarström V, Ljungman P. Immunity to poliovirus and immunization with inactivated poliovirus vaccine after autologous bone marrow transplantation. Clin Infect Dis 1994;18:547-52.

12. Parkkali T, Stenvik M, Ruutu T, Hovi T, Volin L, Ruutu P. Randomized comparison of early and late vaccination with inactivated poliovirus vaccine after allogeneic BMT. Bone Marrow Transplant 1997;20:663-8.

13. Parkkali T, Käyhty H, Ruutu T, Volin L, Eskola J, Ruutu P. A comparison of early and late vaccination with Haemophilus influenzae type $\mathrm{b}$ conjugate and pneumococcal polysaccharide vaccines after allogeneic BMT. Bone Marrow Transplant 1996;18:961-7.

14. Ljungman P, Fridell E, Lönnqvist B, et al. Efficacy and safety of vaccination of marrow transplant recipients with a live attenuated measles, mumps, and rubella vaccine. J Infect Dis 1989;159:610-5.

15. Pauksen K, Duraj V, Ljungman $P$, et al. Immunity to and immunization against measles, rubella and mumps in patients after autologous bone marrow transplantation. Bone Marrow Transplant 1992;9:427-32.

16. Engelhard D, Handsher R, Naparstek E, et al. Immune response to polio vaccine in bone marrow transplant recipients. Bone Marrow Transplant 1991;8:295-300.

17. Vance E, George S, Guinan EC, et al. Comparison of multiple immunization schedules for Haemophilus influenzae type b-conjugate and tetanus toxoid vaccines following bone marrow transplantation. Bone Marrow Transplant 1998;22:735-41.

18. Barra A, Cordonnier C, Preziosi MP, et al. Immunogenicity of Haemophilus influenzae type $\mathrm{b}$ conjugate vaccine in allogeneic bone marrow recipients. J Infect Dis 1992;166:1021-8.

19. Guinan E, Molrine D, Antin J, et al. Polysaccharide conjugate vaccine responses in bone marrow transplant patients. Transplantation 1994;57:677-8.
20. Gandhi MK, Egner W, Sizer L, et al. Antibody responses to vaccinations given within the first two years after transplant are similar between autologous peripheral blood stem cell and bone marrow recipients. Bone Marrow Transplant 2001;28:775-8.

21. Winston DJ, Ho WG, Schiffman G, Champlin RE, Feig SA, Gale RP. Pneumococcal vaccination of recipients of bone marrow transplants. Arch Intern Med 1983;143:1735-7.

22. Saxon A, Mitsuyasu R, Stevens R, Champlin RE, Kimata H, Gale RP. Designed transfer of specific immune responses with bone marrow transplantation. J Clin Invest 1986;78:959-67.

23. Molrine DC, Guinan EC, Antin JH, et al. Haemophilus influenzae type $\mathrm{b}$ (HIB)-conjugate immunization before bone marrow harvest in autologous bone marrow transplantation. Bone Marrow Transplant 1996;17:1149-55.

24. Molrine DC, Guinan EC, Antin JH, et al. Donor immunization with Haemophilus influenzae type $b$ (HIB)-conjugate vaccine in allogeneic bone marrow transplantation. Blood 1996;87:3012-8.

25. Redman RL, Nader S, Zerboni L, et al. Early reconstitution of immunity and decreased severity of herpes zoster in bone marrow transplant recipients immunized with inactivated varicella vaccine. J Infect Dis 1997;176:578-85.

26. Hata A, Asanuma $H$, Rinki $M$, et al. Use of an inactivated varicella vaccine in recipients of hematopoietic-cell transplants. N Engl J Med 2002;347:26-34.

27. National Advisory Committee on Immunization. Approach to vaccination of immunodeficient individuals. In: Canadian Immunization Guide, 6th edn. Ottawa: Canadian Medical Association, 2002:21-30.

28. Ljungman P. Immunization of transplant recipients. Bone Marrow Transplant 1999;23:635-6.

29. Henning KJ, White MH, Sepkowitz KA, Armstrong D. A national survey of immunization practices following allogeneic bone marrow transplantation. JAMA 1997;277:1148-51.

30. Ljungman P, Cordonnier C, deBock R, et al. Immunisations after bone marrow transplantation: Results of a European survey and recommendations from the infectious diseases working party of the European Group for Blood and Marrow Transplantation. Bone Marrow Transplant 1995;15:455-60. 


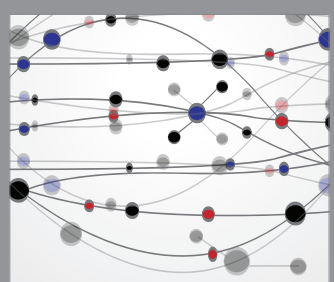

The Scientific World Journal
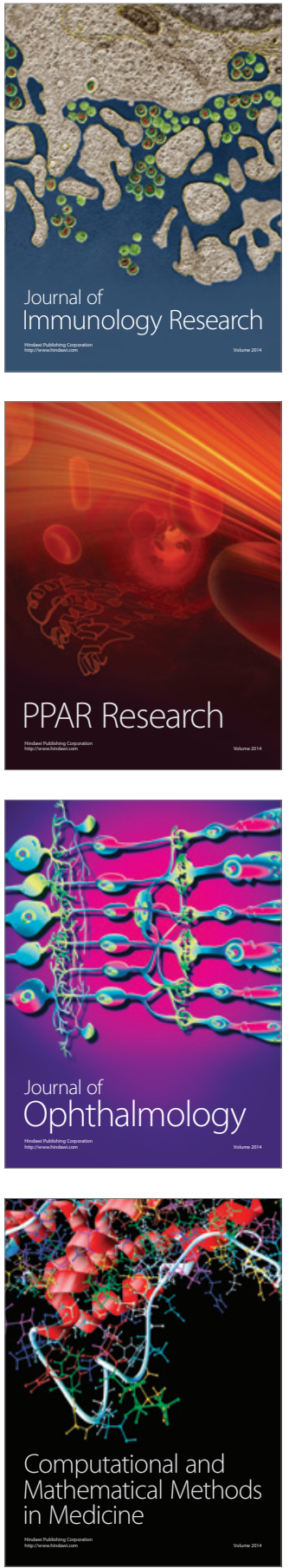

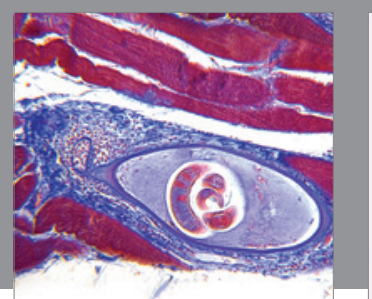

Gastroenterology Research and Practice

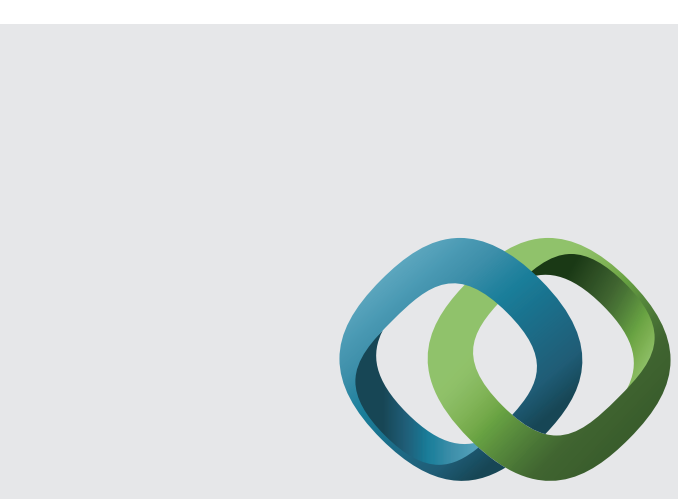

\section{Hindawi}

Submit your manuscripts at

http://www.hindawi.com
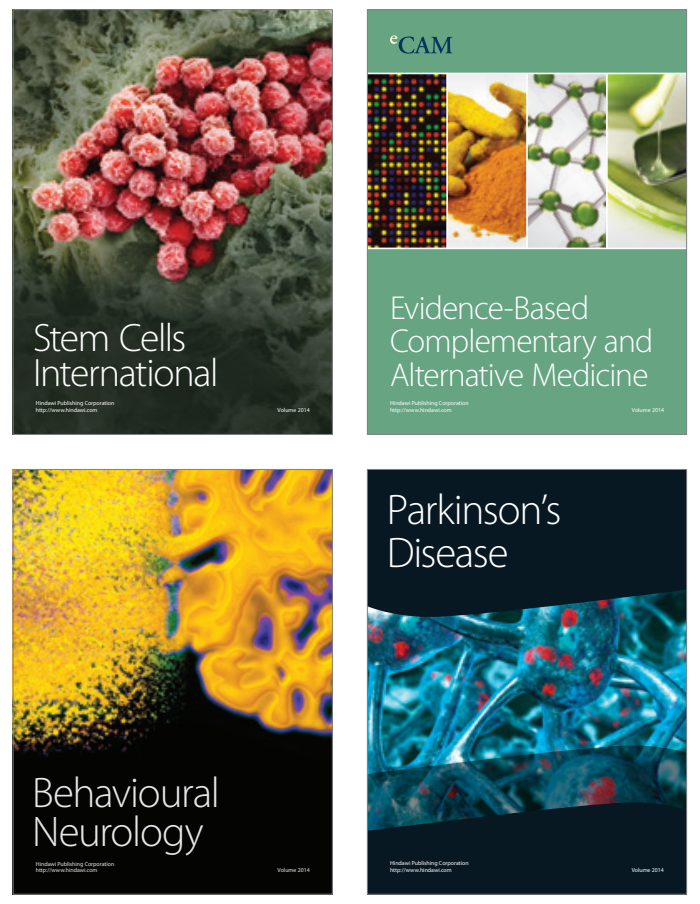
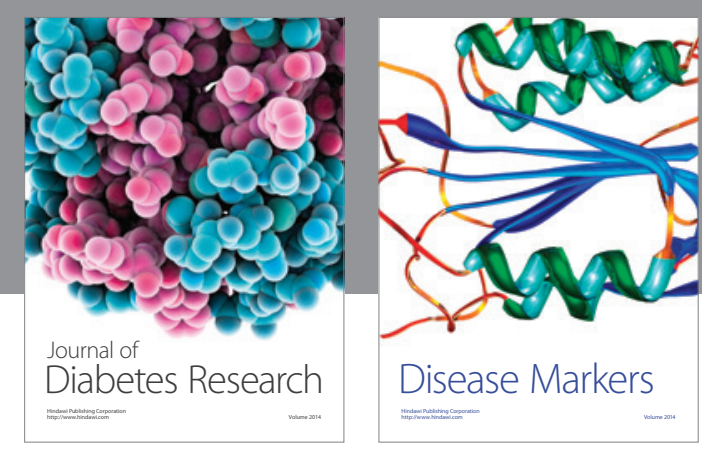

Disease Markers
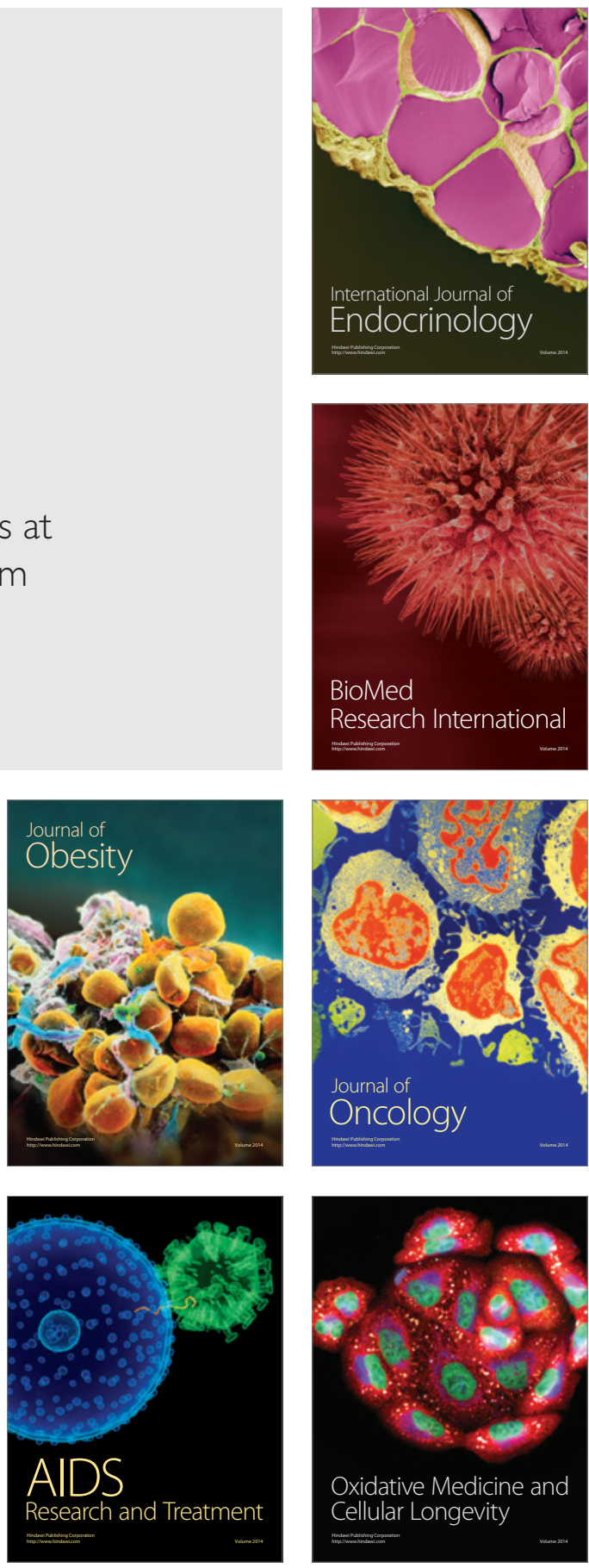